\title{
Precision test for spectral characteristic of NIR spectroscopy and bomb calorimeter for measuring higher heating value of Leucaena Leucocephala
}

\author{
Jetsada Posom ${ }^{1,2}$, Panmanas Sirisomboon ${ }^{3}$, and Wanphut Saechua ${ }^{3, *}$ \\ ${ }^{1}$ Department of Agricultural Engineering, Faculty of Engineering, Khon Kaen University, Khon Kaen, 40002, Thailand \\ ${ }^{2}$ Applied Engineering for Important Crops of the North East Research Group, Department of Agricultural Engineering, Faculty \\ of Engineering, Khon Kaen University 40002, Thailand \\ ${ }^{3}$ Agricultural Engineering Department, Faculty of Engineering, King Mongkut's Institute of Technology Ladkrabang, Bangkok \\ 10520, Thailand
}

\begin{abstract}
The higher heating value (HHV) plays a significant role in determining of the energy potential of biomass. The method to receive this value using a bomb calorimeter takes a long time approximately of 15 minutes. The implementation of near infrared spectroscopy (NIR) technique by generating a prediction model can be replaced the former method in order to reduce time into 2 minutes. Therefore, the precision of both techniques have to be concerned in order to get the reliable values. The repeatability and reproducibility processes were carried out to evaluate the precision of both methods. It was found that the percentage difference of standard deviation (SD) from the mean of repeatability for the NIR was less than 5 $\%$ and for reproducibility was slightly over $5 \%$ (but not over than $10 \%$ ). The SD of repeatability and reproducibility of the bomb calorimeter were $54.6 \mathrm{~J} / \mathrm{g}(0.3 \%$ difference of average HHV value) and $81.3 \mathrm{~J} / \mathrm{g}$ $(0.5 \%$ difference of average HHV value) respectively. The average HHV from the bomb calorimeter was $18,166.77 \pm 236.29 \mathrm{~J} / \mathrm{g}$. Both techniques showed very low percentage difference of the SD from the mean that revealed very high reliability and precision of measurements.
\end{abstract}

\section{Introduction}

The continuously decreasing in fossil fuel leads to finding of alternative energy which can be used instead of conventional energy. The renewable energy such as biomass, wind, geothermal, solar, waves, tides, and hydroelectric power recently plays important role in the alternate energies. Thailand, one of the agricultural countries has also desired in those energy resources. The department of alternative energy development and efficiency (DEDE) has now given an impetus to grow the energy plants in order to use as biomass energy. Leucaena leucocephala is one of the energy plants that have been promoted due to fast growing, good disease resistance, and high potential energy in combustion process. Not only it can be used as an alternative energy, but also can be utilised as wood furniture work. Traditionally, the trade of biomass as wood chip or saw dust used moisture content in materials for indicating selling price. However, higher heating value (HHV) of the biomass better represents potential and capacity of energy contained in the biomass. The agricultural wastes such as corn straw, rice straw, rice husks, and palm kernel also can be used as biomass fuel. Their HHV were 18,195, 15,511, 15,986, and 19,405 J/g respectively[1]. The conventional method (bomb calorimeter) to determine this value takes a long time approximately of 15 minutes per sample excluding preparing process. Using the NIR technique to evaluate the HHV may be a better way to evaluate the HHV of biomass due to shorter time approximately of 2 minutes and easier procedure. However, the NIR technique also requires reference data used for generating the model to predict value based on those references. It was clearly seen that the conventional method took longer time than the NIR method. However, the accuracy of the model created by the NIR scanning requires piles of data and wide and variety range of them. This may take times and attempts to collect data in order to get the robust model but it subsequently saves time and money in long term. There were many researchers done experiments with the NIR scanning to generate model for prediction some specific values or properties of organic substances such as foods, fruits, beverages, and biomass[2-6]. The NIR technique has advantages as non-destructive method, easy procedure, short time process, low cost, environmental friendly and provides precision prediction[7]. Therefore, the procedure before getting the reference data has to be more concerned. The repeatability and reproducibility processes have been claimed and accepted to use to confirm the precision and reliable of the test [8-10]. In this study aimed to reveal

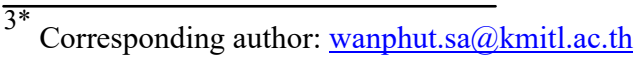


the precision test of both instruments (the NIR spectrometer and the bomb calorimeter) in order to diagnose possible error and to confirm the reliability of the measurements. Additionally, the HHV of Leucaena leucocephala was measured in order to create the model to predict the HHV in future.

\section{Materials and Methods}

The experiments implemented of two instruments which were the NIR spectrometer and the bomb calorimeter with using Leucaena Leucocephala as a sample of biomass. The repeatability and reproducibility were carried out on both techniques.

\subsection{Samples}

Leucaena Leucocephala ranged of $8 \pm 1 \mathrm{~cm}$ dimeter used in this study was 120 samples which were 40 samples from each three parts of Leucaena Leucocephala (lower trunk, middle trunk and upper trunk). Next, they were cut and chopped into small pieces as wood chips by using a chopper machine (P5508, Patipong, Thailand). All samples were dried in an oven at $105^{\circ} \mathrm{C}$ for 24 hours and left in room temperature 24 hours for moisture equilibrium. The repeatability and reproducibility samples were randomly selected of 10 sets of samples for the precision test of bomb calorimeter experiments with making in a pellet shape approximately range of 0.5-1.0 gram. The other random of two sample sets for the precision of the NIR scanning used dried Leucaena Leucocephala chips. The feature of samples was shown in Figure 1.
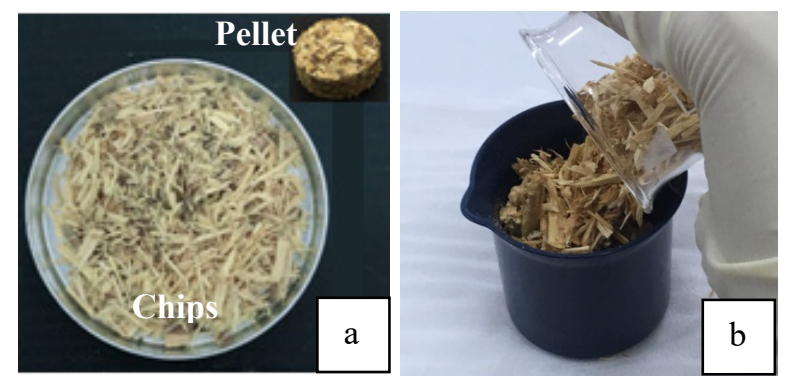

Fig. 1. a) A sample used for the bomb calorimeter b) A sample used for the NIR spectrometer

\subsection{Instruments}

The NIR spectrometer used for scanning was FT-NIR (MPA, Bruker, Germany) in wavenumber range of $12,500-3,600 \mathrm{~cm}^{-1}$ at $8 \mathrm{~cm}^{-1}$ resolution. The bomb calorimeter (C200, IKA, Germany) was used to measure of higher heating values which was divided into two parts each measurement done with 2 replicates per a sample set. The first part was carried out for repeatability and reproducibility, and the second part was determining the average HHV. The calibration process before measuring was carried out in the sample vessel using pelletized benzoic acid (IKA C 723, IKA, Germany).

\subsection{Repeatability and reproducibility}

Repeatability is a method to show the precision of the instrument when the measurement was replicated many times at the same condition. The repeatability process of the NIR was done by scanning the same sample in a quartz cup for 10 times at the same position. Then absorbance values of the same of three wavenumbers $\left(4011.4,5191.7\right.$ and $\left.6800.2 \mathrm{~cm}^{-1}\right)$ of each spectrum were used to determine the average absorbance and the standard deviation values. Reproducibility of the NIR presents the homogenous of the sample. The method was carried out by scanning the same sample and re-loading this sample for 10 times. The three wavenumbers were chosen to evaluate the average absorbance and the standard deviation values.

The repeatability of the bomb calorimeter also presented the precision of the HHV measurements. It was done by combustion of the 10 sample sets with two replications for each set. The differences of higher heating value (HHV) of each sample set were determined and the standard deviation of the differences was calculated. The reproducibility of the bomb calorimeter shows the precision of the process whether different researchers did the experiment the results still accuracy. It was carried out by using of 10 blinded samples compared with their reference values. The different HHV between blinded samples and reference values and the standard deviation of the differences were determined.

The interpretation of the repeatability and reproducibility was consideration of the value of the SD. Typically the accepted SD value depends on the acceptance level for each experiment and normally using not much than $5 \%$ difference from the mean $[11,12]$. The lower SD presented higher precision of the measurement.

\section{Results and Discussions}

The average HHV of Leucaena Leucocephala from the bomb calorimeter showed in Table 1. The average HHV was $18,166.07 \pm 236.29 \mathrm{~J} / \mathrm{g}$ which showed a good energy potential of biomass compared with other agricultural wastes[13] and has an excellent potential for using as combustion fuel as well as saw dust or wood pellet[14]. Additionally, it was found that the different position of Leucaena Leucocephala had no effect on the HHV by analysing with statistical method one way ANOVA at confident level of 95 percentages (Table 1). Figure 2 showed average raw spectra of NIR scanning for 120 samples of Leucaena Leucocephala which consisted peaks of cellulose at wavenumber $6,808 \mathrm{~cm}^{-1}$, water at wavenumber $5,188 \mathrm{~cm}^{-1}$, starch at wavenumber 4,740 $\mathrm{cm}^{-1}$, water at wavenumber $4,277 \mathrm{~cm}^{-1}$, and benzene at wavenumber $3,980 \mathrm{~cm}^{-1}$.

The experiments for repeatability and reproducibility of the NIR scanning and the bomb calorimeter were 
shown in Table 2 and Table 3 respectively. The average HHV of Leucaena leucocephala in repeatability and reproducibility processes from the bomb calorimeter were $17,943.7 \mathrm{~J} / \mathrm{g}$ and $18,208.0 \mathrm{~J} / \mathrm{g}$ respectively. It was found that the NIR spectrometer had an excellent capability for scanning which could notice from very low standard deviation values of repeatability and reproducibility procedures.

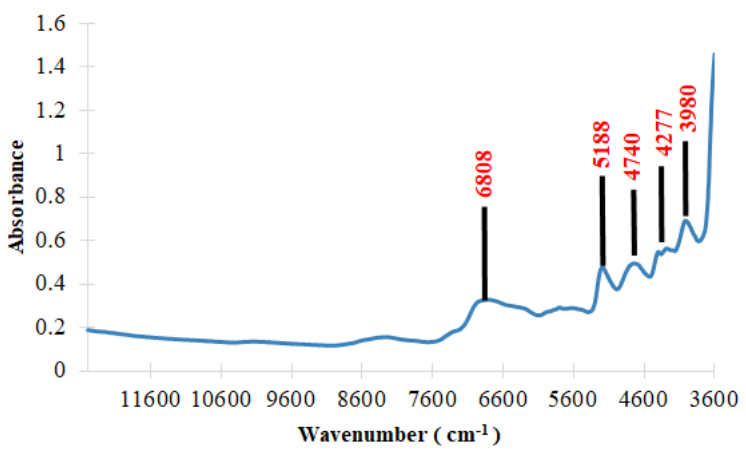

Fig. 2. The average of raw spectra of Leucaena leucocephala

The percentage difference of the SD of the NIR in repeatability showed lower than 5\% compared with the mean of each wavenumber which revealed of precision and reliability of the NIR spectrometer. It was noticed that the percentage difference of the SD of the NIR in reproducibility showed a little higher than $5 \%$ in wavenumber of $5191.7 \mathrm{~cm}^{-1}$ and $6800.2 \mathrm{~cm}^{-1}$ which were $6 \%$ and $7 \%$ respectively. This showed that the sample may be nonhomogeneous if it relied on the accepted level of $5 \%$ difference form the mean. However, this research could imply that the sample had homogenous due to acceptance of the percentage difference of SD value which was not higher than $10 \%$. The reason was the samples could not be sieved because it had to be used like receiving from the chopper process as the commercial trade. This meant that the NIR Spectrometer had ability to replicate the measurement and the samples used in scanning were homogenous.

Table 1. The HHV of Leucaena Leucocephala

\begin{tabular}{|c|c|}
\hline Position & Avg. HHV \pm SD (J/g) \\
\hline Lower trunk & $18,168.28 \pm 243.93^{\mathrm{NS}}$ \\
\hline Middle trunk & $18,182.15 \pm 226.67^{\mathrm{NS}}$ \\
\hline Upper trunk & $18,147.78 \pm 238.28^{\mathrm{NS}}$ \\
\hline $\begin{array}{c}\text { Overall Avg. } \\
\text { HHV } \pm \text { SD }\end{array}$ & $18,166.07 \pm 236.29$ \\
\hline
\end{tabular}

Consistently, the repeatability and reproducibility of the bomb calorimeter showed very low of the standard deviation. The SD of repeatability was $54.6 \mathrm{~J} / \mathrm{g}$ which was approximately of 0.3 percentage of the average HHV from repeatability procedure. The SD of reproducibility was $81.3 \mathrm{~J} / \mathrm{g}$ which was approximately of
0.5 percentage of the average HHV of reproducibility process. This meant that the bomb calorimeter also had ability to replicate measuring and different performers had no effect on measurements. These results of repeatability and reproducibility for both instruments showed very low standard deviations could be helped in analysis process of the prediction in term of the variance of data or the out liner of some spectra could not come from the error of instruments. If there will some errors occur in experiments, the performer have to focus on other causes of which do not from instruments.

Table 2. The repeatability and reproducibility of

\begin{tabular}{|c|c|c|c|c|}
\hline \multirow{2}{*}{$\begin{array}{l}\text { Wave- } \\
\text { number } \\
\left(\mathrm{cm}^{-1}\right)\end{array}$} & \multicolumn{2}{|c|}{ Repeatability } & \multicolumn{2}{|c|}{ Reproducibility } \\
\hline & $\begin{array}{l}\text { Avg. } \\
\text { Abs. } \\
\pm \text { SD }\end{array}$ & $\begin{array}{l}\% \\
\text { different } \\
\text { from the } \\
\text { mean }\end{array}$ & $\begin{array}{l}\text { Avg. } \\
\text { Abs. } \\
\pm \text { SD }\end{array}$ & $\begin{array}{l}\% \\
\text { different } \\
\text { from the } \\
\text { mean }\end{array}$ \\
\hline 4011.4 & $\begin{array}{l}0.6705 \pm \\
0.0008\end{array}$ & 0.1 & $\begin{array}{l}0.7256 \pm \\
0.0318\end{array}$ & 4.4 \\
\hline 5191.7 & $\begin{array}{l}0.4586 \pm \\
0.0010\end{array}$ & 0.2 & $\begin{array}{l}0.5153 \pm \\
0.0299\end{array}$ & 5.8 \\
\hline 6800.2 & $\begin{array}{l}0.3114 \pm \\
0.0012\end{array}$ & 0.4 & $\begin{array}{l}0.3626 \pm \\
0.0262\end{array}$ & 7.2 \\
\hline
\end{tabular}

Table 3. The repeatability and reproducibility of the bomb calorimeter.

\begin{tabular}{|c|c|c|}
\hline \multirow{2}{*}{ Samples } & Repeatability & Reproducibility \\
\hline & $\begin{array}{c}\text { The difference of } \\
\text { HHV }(\mathrm{J} / \mathrm{g})\end{array}$ & $\begin{array}{c}\text { The difference of } \\
\text { HHV }(\mathrm{J} / \mathrm{g})\end{array}$ \\
\hline 1 & 173.0 & 184.0 \\
\hline 2 & 127.0 & 288.0 \\
\hline 3 & 35.0 & 71.0 \\
\hline 4 & 14.0 & 100.5 \\
\hline 5 & 54.0 & 241.5 \\
\hline 6 & 149.0 & 187.5 \\
\hline 7 & 70.0 & 42.5 \\
\hline 8 & 54.0 & 95.0 \\
\hline 9 & 138.0 & 83.5 \\
\hline 10 & 58.0 & 94.5 \\
\hline $\begin{array}{r}\text { SD of } \\
\text { differences }\end{array}$ & 54.6 & 81.3 \\
\hline
\end{tabular}




\section{Conclusions}

It can be concluded that the NIR spectrometer and bomb calorimeter have an excellent precision and reliability for using to evaluate the HHV of Leucaena Leucocephala. This could help the experiments to be achieved in generating of reliable prediction model. However, the success model generated from the NIR in order to reduce the process time for determining the HHV with the bomb calorimeter requiring more robust model which should be developed with more samples and more variation samples.

\section{Acknowledgements}

Thanks to Near Infrared Spectroscopy Research Centre for Agricultural Product and Food (www.nirsresearch. com), the Department of Agricultural Engineering, Faculty of Engineering, King Mongkut's Institute of Technology Ladkrabang, Thailand.

\section{References}

1. H. Quian, X. Guo, S. Fan, K. Hagos, X. Lu, C. Liu, D. Huang, J. Chem. Eng. 61,12(2016)

2. P. Jetsada, S. Panmanas, Energy.121(2017)

3. P. Jetsada, S. Panmanas, S. Wanphut, Energy.103(2017)

4. P. Jetsada, S. Panmanas, Bio. Eng.130(2015)

5. B.M. Nicolai, B. Katrien, B. Els, P. Ann, S. Wouter, I.T. Karen, L. Jeroen, Postharvest Bio. and Tech.46,2(2007)

6. J.M. Triolo, A.J. Ward, P. Lene, M.L. Mette, Q. Haiyan, G.S. Sven, Appl. Energy. 116(2014)

7. G. Ainara, F.O.V.Silvia, E. Nerea, D. Alberto, M.M. Juan, Trends in Analytical. Chem.76(2016)

8. S. Panmanas, K. Apidul, W. Phil, J. NIR. 21,2(2013)

9. A. Ferragina, C. Cipolat-Gotet, A. Cecchinato, M. Pazzola, M.L. Dettori, G.M. Vacca, G. Bittante, J. Dairy Sci. 100,5(2017)

10. D.M. Musingarabwi, H.H. Nieuwoudt, P.R. Young, H.A. Eyeghe-Bickong, M.A. Vivier, Food Chem.190(2016)

11. J.W. Bartlett, C. Frost, Ultrasound Obstet Gynecol. 31,4(2008)

12. J.M. Bland, D.G. Altman, Lancet. 1,8476(1986)

13. Z. Ke, Z. Ling, B. Michael, X. Feng, Y. Jianming, W. Donghai, Energy. 118(2017)

14. Z. Yaning, G, Xiaoyan, L. Bingxi, Z. Haochun, Q. Baoheng, W. Yifan, Fuel. 159(2015) 